\title{
Seismology of Giant Planets: General Overview and Results from the Kepler K2 Observations of Neptune
}

\author{
Patrick Gaulme $1,2,3, \star$ \\ ${ }^{1}$ Department of Astronomy, New Mexico State University, P.O. Box 30001, MSC 4500, Las Cruces, NM 88003-8001, USA \\ ${ }^{2}$ Apache Point Observatory, 2001 Apache Point Road, P.O. Box 59, Sunspot, NM 88349, USA \\ ${ }^{3}$ Physics Department, New Mexico Institute of Mining and Technology, 801 Leroy Place, Socorro, NM 87801, USA
}

\begin{abstract}
For this invited contribution, I was asked to give an overview about the application of helio and asteroseismic techniques to study the interior of giant planets, and to specifically present the recent observations of Neptune by Kepler K2. Seismology applied to giant planets could drastically change our understanding of their deep interiors, as it has happened with the Earth, the Sun, and many main-sequence and evolved stars. The study of giant planets' composition is important for understanding both the mechanisms enabling their formation and the origins of planetary systems, in particular our own. Unfortunately, its determination is complicated by the fact that their interior is thought not to be homogeneous, so that spectroscopic determinations of atmospheric abundances are probably not representative of the planet as a whole. Instead, the determination of their composition and structure must rely on indirect measurements and interior models. Giant planets are mostly fluid and convective, which makes their seismology much closer to that of solar-like stars than that of terrestrial planets. Hence, helioseismology techniques naturally transfer to giant planets. In addition, two alternative methods can be used: photometry of the solar light reflected by planetary atmospheres, and ring seismology in the specific case of Saturn. The current decade has been promising thanks to the detection of Jupiter's acoustic oscillations with the ground-based imaging-spectrometer SYMPA and indirect detection of Saturn's $f$-modes in its rings by the NASA Cassini orbiter. This has motivated new projects of ground-based and space-borne instruments that are under development. The K2 observations represented the first opportunity to search for planetary oscillations with visible photometry. Despite the excellent quality of K2 data, the noise level of the power spectrum of the light curve was not low enough to detect Neptune's oscillations. The main results from the K2 observations are the clear detection of the well-known differential rotation of Neptune, measured for the first time through the rotational modulation of its photometry, and the detection of the Sun's oscillations, for the first time in an indirect way in intensity measurements.
\end{abstract}

\section{Interior Structure}

The deep internal structures of Jupiter and Saturn, particularly the amount and the radial distribution of heavy elements, are important diagnostics to the formation and evolution of planetary systems in general. Two scenarios are envisioned for the formation of giant planets. The first assumes that cores of rocks and ices are formed by the accumulation of solid planetesimals. When these solid cores grow to about $10 M_{\oplus}$ (Earth masses), a phase of rapid $\mathrm{H}$ He gas accretion follows, which leads to the current structure of the planets [1]. In this class of models, the resulting planets are generally enriched in heavy elements. In the second scenario, giant planets form by gravitational instability of the gas in a massive solar nebula [2], resulting in planets with solar chemical compositions and masses probably larger than that of Jupiter. In this scenario, a solid core may form through sedimentation of solid and vaporized material toward the center, or by subsequent capture of solid planetesimals. The gravitational instability sce-

\footnotetext{
^e-mail: gaulme@nmsu.edu
}

nario requires a relatively massive and cold protoplanetary nebulae to become unstable, while the nucleated instability mechanism can also operate with less massive and hotter disks. Current uncertainties about Jupiter's and Saturn's deep interior structure and composition are large and prevent us from uniquely distinguishing between these two formation mechanisms [3-5].

Jupiter and Saturn are natural reservoirs of liquid metallic hydrogen and helium at pressures of up to tens of Mbar and temperatures of the order of $10^{4} \mathrm{~K}$. Such warm, dense states of matter are very challenging to reproduce in laboratory experiments. Megabar pressures can be achieved in shock compression experiments but usually at temperatures well above those found in giant planets [3]. So far, these experiments have not directly probed the conditions of interest for giant planets. On the other hand, significant progress has been achieved recently in computer simulations of the equations of state of $\mathrm{H}$ and He. Oddly, this has not led to a better consensus on the internal structure of Jupiter [4, 5]. 


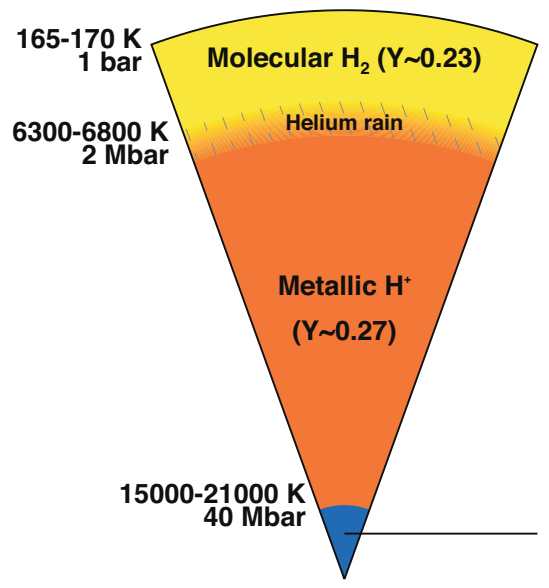

Jupiter

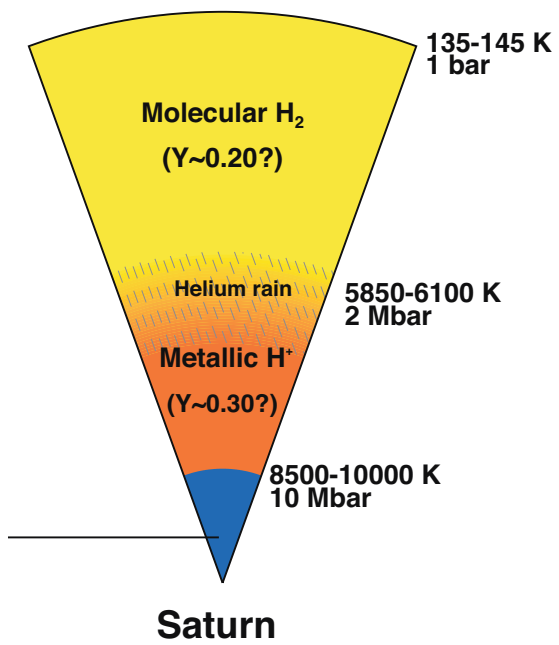

Saturn

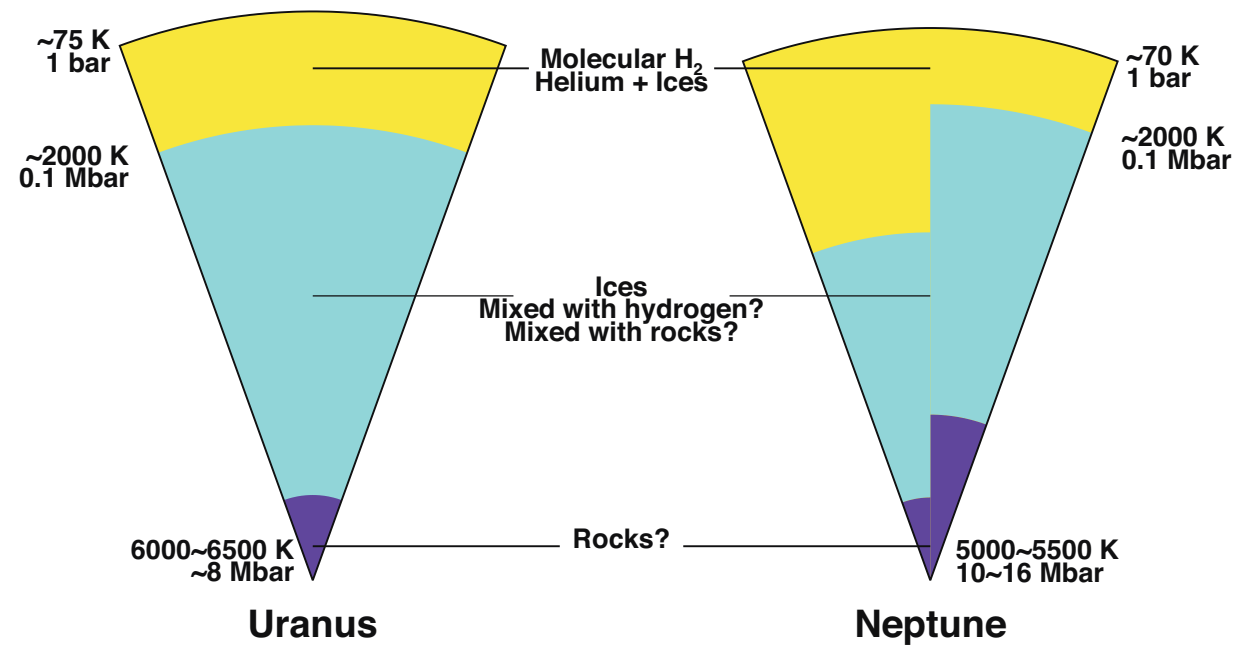

Figure 1: Schematic representation of the interiors of Jupiter, Saturn, Uranus, and Neptune. The range of temperatures for Jupiter and Saturn is for models neglecting the presence of the inhomogeneous region (adapted from [6] and taken from [7]). Helium mass mixing ratios $\mathrm{Y}$ are indicated. The size of the central rock and ice cores of Jupiter and Saturn is very uncertain (see text). Similarly, for Uranus and Neptune, considerable uncertainties exist [8]. Recent models of Neptune indicate a wider range of possible solutions of which two representative ones are shown.

Based on our current knowledge of planetary system formation and of equations of state of dense matter, the debate regarding the internal structure and composition of Jupiter and Saturn can be articulated in terms of four main questions [e.g. 9]. i) What is the radial distribution of heavy elements? Are they concentrated in a central core or distributed throughout the H-He envelope? If mixing processes have redistributed the heavy elements after its formation, the thermal evolution of the planet would be profoundly affected $[10,11]$. ii) If a dense central core is present, what are its mass and composition? Current estimates of the core mass of Jupiter range from 0 to $15 M_{\oplus}$ and the total mass of heavy elements from 10 to $40 M_{\oplus}[3-$ 5]. iii) Which mechanisms dominate the energy transport from the deep interior to the surface of these planets? iv) Do H-He mixtures separate in giant (exo)planets resulting in a depletion of $\mathrm{He}$ in the outer envelope?

\section{Seismology and Giant Planets}

Starting in 2016, NASA's Juno mission will make key contributions to our understanding of Jupiter thanks to precise measurements of its gravity and magnetic fields. Unfortunately, the presence of a denser core of heavy elements only weakly influences even the lowest order (quadrupole) deviation in the gravity field and the core mass will remain essentially model-dependent. On the other hand, seismology, which consists of identifying global acoustic eigenmodes ( $p$-modes), complements Juno science by offering a way to directly measure the planet's sound speed profile, and thus its physical properties from the outer envelope to the core.

All of these questions can be addressed with seismology. From an observational point of view, seismology of giant planets is a natural extension of helioseismology. Their common fluid nature is expected to lead to similar oscillations and to the possibility of using similar observa- 
Table 1: Comparison of physical and seismic parameters for the four giant planets. Asymptotic $\Delta v_{\mathrm{as}}$ are obtained as the inverse of the acoustic travel time across the planet, computed from internal structure models $([3,8,12]$ for Jupiter, Saturn, and Neptune respectively, and Nettelmann priv. comm. for Uranus.). $\Delta v_{\mathrm{sl}}$ and $v_{\max }$ are extrapolated from solar values using asteroseismic scalings with correction of planetary oblateness $e$. For Jupiter, observed values from [13] are shown for comparison. Table from [7].

\begin{tabular}{lcccccccccc}
\hline Planet & $\begin{array}{c}M \\
\left(M_{\mathrm{E}}\right)\end{array}$ & $\begin{array}{c}R_{\mathrm{eq}} \\
(\mathrm{km})\end{array}$ & $\begin{array}{c}e \\
(\%)\end{array}$ & $\begin{array}{c}T_{\text {eff }} \\
(\mathrm{K})\end{array}$ & $\begin{array}{c}\Delta v_{\mathrm{as}} \\
(\mu \mathrm{Hz})\end{array}$ & $\begin{array}{c}\Delta v_{\mathrm{sl}} \\
(\mu \mathrm{Hz})\end{array}$ & $\begin{array}{c}\Delta v_{\text {obs }} \\
(\mu \mathrm{Hz})\end{array}$ & $\begin{array}{c}v_{\text {max,sl }} \\
(\mu \mathrm{Hz})\end{array}$ & $\begin{array}{c}v_{\text {max obs }} \\
(\mu \mathrm{Hz})\end{array}$ & $\begin{array}{c}\delta v_{\text {rot }} \\
(\mu \mathrm{Hz})\end{array}$ \\
\hline Sun & $310^{5}$ & 798000 & 0 & 5777 & 138 & & & & 3100 & 0.4 \\
\hline Jupiter & 317.9 & 71492 & 6.5 & 125 & $152-156$ & 134 & 155 & 2030 & 1250 & 28 \\
Saturn & 95.1 & 60268 & 10.2 & 95 & $111-115$ & 99 & & 1040 & & 26 \\
Uranus & 14.53 & 25559 & 2.4 & 57 & $\sim 172$ & 130 & & 1030 & & 17 \\
Neptune & 17.14 & 24764 & 2.7 & 59 & $198-213$ & 148 & & 1270 & & 19 \\
\hline
\end{tabular}
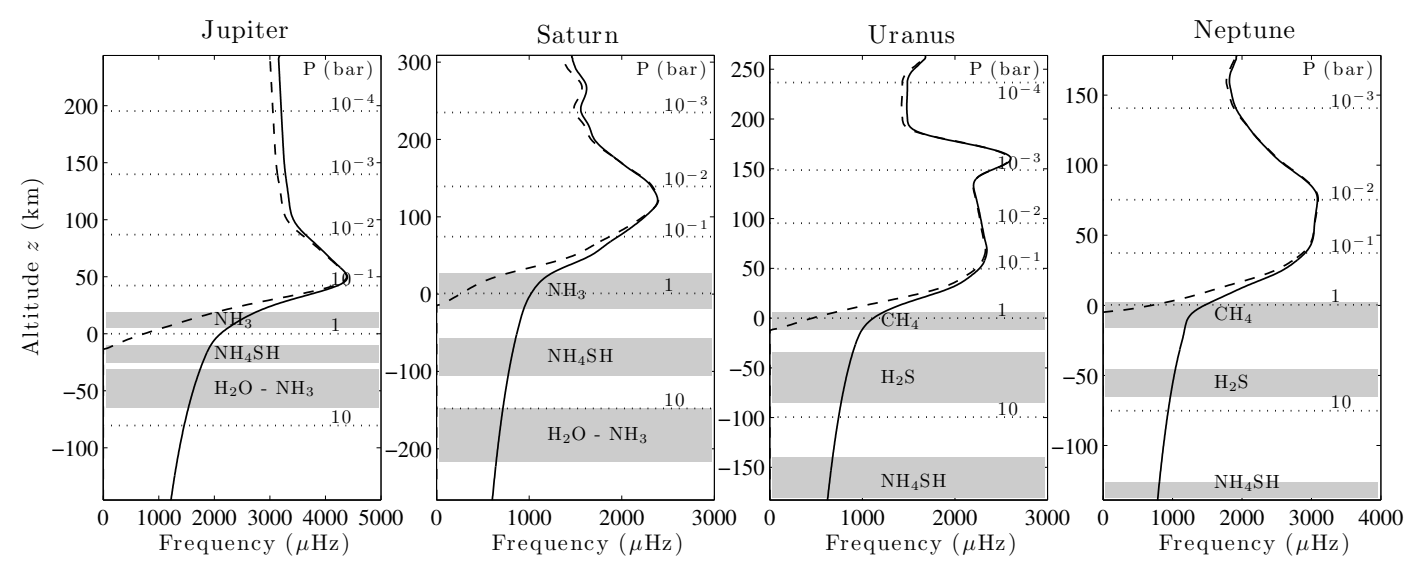

Figure 2: Acoustic cut-off $v_{\mathrm{c}}$ (plain line) and Brünt-Väisälä $N / 2 \pi$ (dashed line) frequency profiles in upper atmospheres of the four giant planets. Grey regions indicate cloud locations and their chemical compositions [e.g. 14]. Above the 1-bar level $(z \equiv 0 \mathrm{~km})$, pressure-temperature profiles come from radio occultation of Voyager spacecrafts [15, and references therein]. Below $z=0$, pressuretemperature profiles are adiabats, which imposes $N=0$. From [7].

tional techniques. Theoretical works $[16,17]$ predict that Jovian global oscillations should have a frequency range of $[0.8,3.5] \mathrm{mHz}$ with 10 to $100 \mathrm{~cm} \mathrm{~s}^{-1}$ amplitude, values that are comparable to those of the Sun. In Table 1, we report the physical and seismic parameters for the four giant planets. Especially, we include the estimates of frequency at maximum amplitude $v_{\max }$ and mean frequency spacing between mode overtones $\Delta v$ obtained from the asteroseismic scaling relations. Indeed, even though giant planets present significant differences with respect to stars, we still expect $v_{\max }$ to be proportional to the acoustic cut-off frequency (a function of local gravity and pressure scale height), and $\Delta v$ to the square root of the mean density of the body. The acoustic cut-off vertical profile of the four giant planets is represented is Fig. 2, compared with the location of cloud and haze layers. For Jupiter and Saturn, waves are similarly trapped close to the ammonia cloud decks, even though Saturn's cut-off is about half Jupiter's. For Uranus and Neptune, waves are trapped at the base of the methane clouds, by keeping in mind that the extension and thickness of cloud coverage suffers from strong uncertainties. We also represent the Brünt-Väisälä frequency profile, which displays values similar to the acoustic cut- off, which means that coupling between acoustic and gravity waves may exist above the tropopause.

Observationally, most of the efforts dedicated to the search of oscillations of giant planets regarded Jupiter, as it is the biggest, closest, and brightest target. There have been several attempts to detect Jovian oscillations using infrared photometry [18], Doppler spectrometry [19-21], and careful searches for excitation of acoustic waves due to the impact of the Shoemaker-Levy 9 comet [22, 23]. In most of these campaigns, the signal-to-noise (SNR) ratio was too low or instrumental artifacts were present that inhibited any positive detection. The fast rotation of Jupiter also limits the precision these instruments were able to obtain.

Jovian seismology had to wait until 2011 to get the first strong evidence of the detection of oscillations using the SYMPA instrument, an imaging spectrometer based on a Mach-Zehnder interferometer at fixed optical path difference [24]. This instrument was designed to overcome some of the earlier limitations by imaging the full planetary disk, similar to solar helioseismic instruments like GONG [25], MDI/SOHO [26], and HMI/SDO [27]. As part of a 10-day observing run in 2005, the SYMPA instrument was able to produce a power spectrum of Jupiter's 


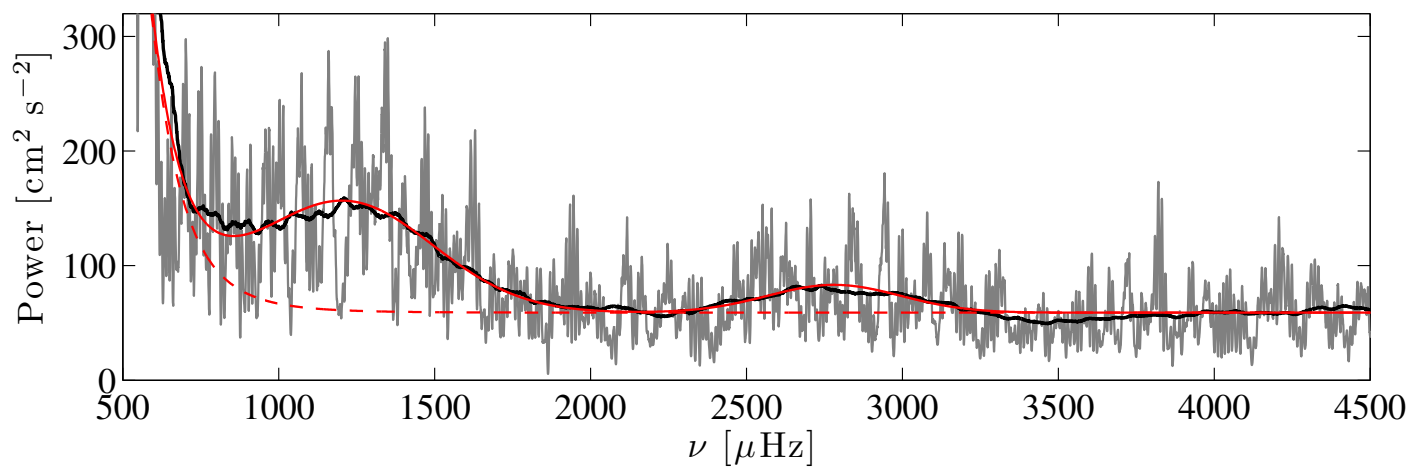

Figure 3: Evidence of the first detection of Jupiter's global modes from the SYMPA instrument, similar to the one to be built in this project. It shows the power spectrum of the mean velocity time series obtained in 2005. Excess oscillation power is detected between 800 and $3400 \mu \mathrm{Hz}$, as well as a comb-like structure of regularly spaced peaks. The thick lines are smoothed data. From [13].

oscillations shown in Fig. 3 [13, 28]. An excess of acoustic power is observed in the frequency range predicted by theory, as well as the comb-like structure of peaks that is also expected from interior models, thereby confirming Jupiter's global pulsations. Unfortunately, the level of noise in the data is too high to identify individual modes and decisively probe Jupiter's interior.

Regarding Saturn, Marley \& Porco (1993) [29] envisionned that "the rings of Saturn may act as a seismograph, recording gravitational perturbations associated with acoustic oscillations modes of the planet". The basic idea is that wave features in Saturn's $C$ rings could be created by resonant interactions with fundamental $(f)$ oscillation modes (i.e., radial order $n=0$ ), since these modes perturb the internal density profile and, therefore, the external gravity field. Twenty years later, observations of stellar occultations of stars by the rings made with Cassini showed that density-wave structures detected in the C-ring were compatible with resonances due to Saturn $f$-modes [30-32]. These observations are the indirect evidence of these wave forcings [32]. However, observations unexpectedly displayed a splitting of the oscillation frequencies for several azimuthal orders. In a first attempt to explain this, and to retrieve information about the Physics of Saturn's interior, Fuller (2014) [31] proposed that a thermally stable stratified layer might surround the core. This would lead to the existence of a gravity $(g)$ mode cavity, and these $g$-modes would interfere with Saturn's $f$-modes and cause the observed split frequencies, somewhat like $g$-modes do with $p$-modes in red-giant stars [33].

Seismology of giant planets looks similar to asteroseismology in the 1990s, i.e. with a bright future. The current decade has been promising thanks to the detection of Jupiter's acoustic oscillations with the ground-based imaging-spectrometer SYMPA, and indirect detection of Saturn's $f$-modes in its rings by the NASA Cassini orbiter. This has motivated new projects of ground-based instruments that are under development, such as the multisite imaging spectrometer JOVIAL/JIVE (Gonçalves et al., submitted), inherited from SYMPA, which is led by teams from the Observatoire de la Côte d'Azur and New
Mexico State University. In the following, we discuss the NASA Kepler "K2" mission, which observed Neptune for about 49 days in early 2015. It also observed Uranus in 2016, but data are not processed yet. Even though it is not sure whether visible photometry is appropriate for giant planet seismology, it was a unique opportunity to test this technique.

\section{The Visible Photometry Approach}

Several approaches can be used for detecting acoustic modes of giant planets: visible photometry for measuring reflected solar flux changes, infrared photometry for temperature fluctuations, and Doppler spectrometry for radial velocities. The choice of an observation technique is also tightly bounded to the choice of a spectral domain. From the visible up to wavelengths of 5 to $20 \mu \mathrm{m}$, the spectra of the four giants is dominated by the solar spectrum that is reflected by clouds and hazes, on top of which are superimposed absorption lines of atmospheric constituents (e.g. $\mathrm{H}_{2}, \mathrm{CH}_{4}, \mathrm{NH}_{3}$ ). At larger wavelengths, in the mid infrared, their spectra result from thermal emission. The thermal spectrum dominates the reflected spectrum starting at about $6.5 \mu \mathrm{m}$ for Jupiter, $10.6 \mu \mathrm{m}$ for Saturn, 18 $\mu \mathrm{m}$ for Uranus, and $15 \mu \mathrm{m}$ for Neptune. From Wien's law, their peak emissions are $23 \mu \mathrm{m}$ for Jupiter, $30 \mu \mathrm{m}$ for Saturn, and $49 \mu \mathrm{m}$ for Uranus and Neptune. Obviously, whatever the technique, seismic observations of giant planets follow the same rules as asteroseismic observations: high duty cycle, and long duration (at least 1 week) to reach a frequency resolution sufficient for identifying modes.

A natural approach to perform seismology of the giant planets is to search for variations of the solar light reflected by Jupiter, resulting from distortions of the planetary external radius $R$ by acoustic modes. Mosser (1995) [34] shows that a radial mode $l=0$ of velocity $v$ leads to a variation of the radius $\delta R=c_{\mathrm{s}} v / g$, where $c_{\mathrm{s}}$ is the sound speed in the upper troposphere and $g$ the gravitation. For Jupiter, $c_{\mathrm{s}} \simeq 800 \mathrm{~m} \mathrm{~s}^{-1}, g=24.8 \mathrm{~m} \mathrm{~s}^{-2}$, so that a $1-\mathrm{m} \mathrm{s}^{-1}$ velocity implies $\delta R=40 \mathrm{~m}$, with a corresponding variation of reflected luminosity, $(\delta \Phi / \Phi)_{\text {refl }}=2 \delta R / R$ of the order of 
$1 \mathrm{ppm}$. We expect about the same level for the four giant planets.

Regarding the photometric approach, Gaulme \& Mosser (2005) [35] showed that the photometric fluctuations related to planetary radius variations are likely to be negligible with respect to albedo fluctuations of clouds that are caused by acoustic modes on their thermodynamical equilibrium. They studied Jupiter's cloud response to pressure-temperature perturbations, as acoustic waves, with a one-dimensional model including the thermodynamics and microphysics in a single cloud layer. They demonstrated that acoustic waves drive a shift in the gas/condensate phase equilibrium that defines the cloud, which is damped by the kinetics of condensation. The resulting perturbation of the cloud equilibrium can be converted to albedo variations. For acoustic waves of $1-\mathrm{m} \mathrm{s}^{-1}$ amplitudes, photometric fluctuations reach several tens of ppm, which comfortably fits within the photometric performance of current space-based imagers.

The Gaulme \& Mosser results are model dependent, and strongly rely on our knowledge of cloud properties of the giant planets. Therefore the photometric approach looks at the same time both promising and uncertain for a space mission. On the one hand, photometric imaging instruments are much simpler and lighter than highprecision spectrometers and their feasibility and accuracy has been demonstrated by the remarkably successful asteroseismic observations of CoRoT and Kepler [36, 37]. Note that such an approach looks more suitable for Jupiter and Saturn rather than for Uranus and Neptune, because a clear cloud structure dominates the reflected solar light of gas giants, while the cloud coverage of ice giants is sparse at levels where sunlight is reflected. On the other hand, the mission K2 represented an exceptional opportunity of testing this technique, because it was mostly free. No much risks and only new information to grab, both in case of detection or non-detection of planetary oscillations. However, K2 could focus only on the ice giants, as Jupiter and Saturn would saturate and move too fast on the chip to allow for precise photometric measurements.

\section{The K2 Observations}

\subsection{Light Curve}

Starting on December 1st 2014, Kepler K2 observed Neptune continuously for 49 days at a 1-minute cadence. The processing of $\mathrm{K} 2$ photometric light-curves is reported by Rowe et al. (submitted). It includes corrections for photometric jumps, intrapixel variations and outliers. The light curve was detrended to remove the observed decrease in flux due to the increasing distance between Neptune and the Kepler spacecraft, by subtracting a second-order polynomial (Fig. 4a). Over the 49-day observation window the distance between the Kepler spacecraft and Neptune increased by $0.81 \mathrm{AU}$, which represents a 406-second variation of light travel time. Since we consider physical phenomena on Neptune and on the Sun, we interpolated the data onto a uniform time grid that takes into account the
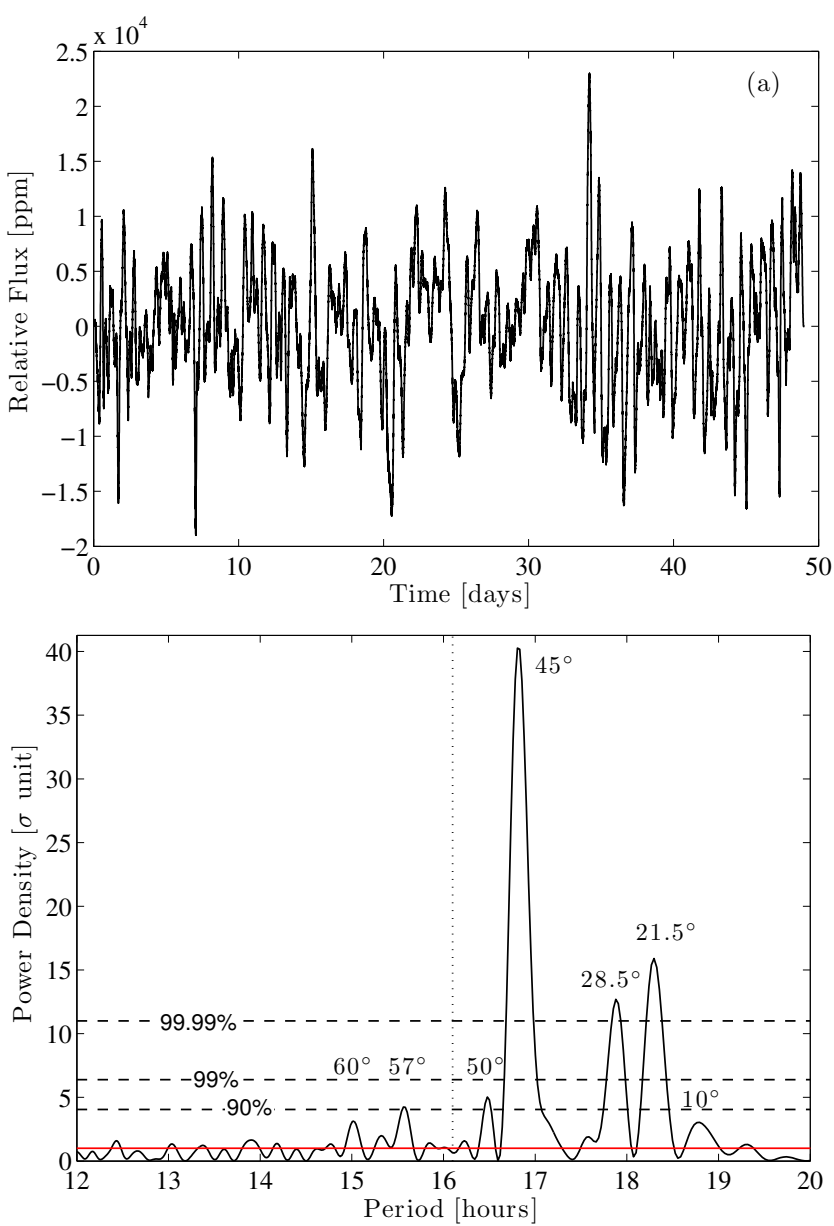

Figure 4: Panel a: K2 Neptune full 49-day light curve, showing normalized brightness variations over time elapsed since December 1, 2014. Panel b: oversampled and whitened power density spectrum as a function of period. The red line corresponds to the noise level of the whitened spectrum and black dashed lines indicate statistical significance levels. Numbers above some peaks indicate the latitudes on Neptune corresponding to that rotation period based on the zonal velocity curve given by SánchezLavega et al. (2015); the features could be in either hemisphere. From [38].

light travel time. The distance variation from the Sun to Neptune is negligible as it corresponds to 0.8 seconds.

The light curve was analyzed in terms of frequencies by computing its power spectral density (PSD) with a Fast Fourier Transform. All short gaps (only a few missing points) were interpolated with a second-order polynomial estimated from the nearby data points. There are no long gaps observed and the overall duty cycle is greater than $98 \%$. Simon et al. (2016) [38] reported the clear detection of three groups of dominant peaks in between 15 and $17 \mu \mathrm{Hz}, 30$ and $33 \mu \mathrm{Hz}$, and 45 and $50 \mu \mathrm{Hz}$, corresponding to Neptune's rotation and two of its harmonics. Figure 4 shows the periods corresponding to Neptune's rotation (15-17 $\mu \mathrm{Hz}$ region). The three major peaks are undoubtedly signal (confidence level larger than 99.99\%), while the neighboring peaks are marginally detected with confidence levels around $90 \%$ (in between $3 \sigma$ and $6 \sigma$ ). None 
of the peaks correspond to the periods of Neptune's major moons, nor their harmonics. Horizontal oscillations detected in prior Keck observations [39], potentially linked to tidal forcing by Triton, did not produce a corresponding $7.24 \mathrm{hr}$ signal in our analysis of the photometric light curve. The peaks in the periodogram, if assumed to be created by discrete cloud features, can be used to infer the latitude of those features based on a symmetric zonal wind profile. The most significant peaks roughly correspond to latitudes of $45^{\circ}, 28.5^{\circ}$, and $21.5^{\circ}$ planetographic latitude, respectively. Since the wind profile is symmetric around the equator, these results cannot distinguish between northern or southern features, and we neglect any dispersion in the zonal velocities for the moment. The hemispheric degeneracy can be broken with direct imaging observations of Neptune's cloud locations.

Simultaneous KECK observations allowed to infer that the main feature at $45^{\circ}$ is a very bright spot located in the southern hemisphere, which has been observed many times (e.g., [40]). For some smaller features closer to the equator the orientation of the planet make it harder to determine the hemisphere. The dramatic bright spot is long lived and is responsible for the principal component of the variation over a single rotation. However, multiple smaller features both produce irregularities in the light curve and seem to evolve over more rapid timescales, at time as quickly as within a rotation or two, which is compatible with previous observations (e.g., [41]). Without the largest feature, the light curve would be far more irregular, and without the varying smaller spots the rapidity of the evolution would be much less.

\subsection{Search for Neptune Oscillations}

From Fig. 2, Neptune's maximum cut-off frequency for acoustic modes is about $3000 \mu \mathrm{Hz}$. It means that acoustic modes cannot stand in Neptune at frequencies larger than $3000 \mu \mathrm{Hz}$. The possible acoustic waves excited at larger frequencies are not trapped by Neptune's troposphere and get dissipated in the stratosphere. More precisely, after increasing monotonically in the upper troposphere, the cut-off frequency reaches a maximum at the tropopause, and decreases to a plateau at about $2000 \mu \mathrm{Hz}$ in the midstratosphere. As analyzed by Mosser et al. (1995) [34] for Jupiter, it means that waves with frequencies between 2000 and $3000 \mu \mathrm{Hz}$ can leak into the troposphere by tunneling effect, making their trapping less efficient. Regarding the lower limit, the acoustic cut-off profile shows that waves with frequencies less that $800 \mu \mathrm{Hz}$ are trapped deeper than pressure levels of about 10 bars. Even though radiative transfer in these planets is not fully constrained, it is very likely that optical observations do not probe that deep. Wave at frequencies lower than $800 \mu \mathrm{Hz}$ are thus evanescent at altitudes probed by optical observations. It it therefore reasonable to expect Neptune oscillations' maximum amplitude to be between 800 and $2000 \mu \mathrm{Hz}$. Note this is perfectly consistent with the $v_{\max }$ arising from the asteroseismic relations (Table 1).

Regarding the mean frequency spacing between mode overtones, it is expected to range from 198 to $213 \mu \mathrm{Hz}$

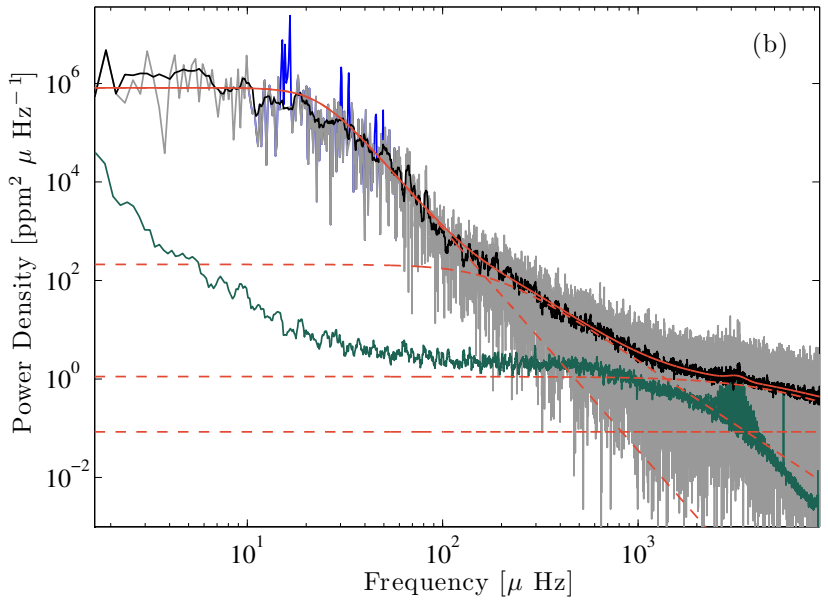

Figure 5: gray line is the power density spectrum of the Kepler light curve in the square of parts per million $\left(\mathrm{ppm}^{2}\right)$ per $\mu \mathrm{Hz}$, as a function of frequency $(\mu \mathrm{Hz})$. Blue peaks are Neptune's rotation frequencies and harmonics. Black line is the power density smoothed over 100 bins to guide the eye to the mean noise level. The plain red line indicates the noise model plus the mode envelope, which is the sum of three semi-Lorentzians, a Gaussian, and a white noise offset (dashed red lines). The excess power due the solar modes is visible in the bottom right of the plot. The green line is the smoothed (100 bins) power density spectrum of the VIRGO/SPM light curve taken simultaneously with K2 data. From [42].

according to internal structure models [7]. This is quite far from the value provided by the asteroseismic scaling relation, likely because of the planet's internal density discontinuities, which affect the distribution of oscillation frequencies. In addition, because of Neptune's fast rotation, acoustic modes of non-radial oscillations with azimuthal order $m$ larger than zero split apart into $+m$ and $-m$ peaks separated by $m$ times the inverse rotation period, i.e. $\approx m \times 17.4 \mu \mathrm{Hz}$.

The power spectral density (Fig. 5) does not display any excess power typical of global oscillations in the [800, $2000] \mu \mathrm{Hz}$ range, and nowhere else except for the solar oscillations. We also searched for Neptune's oscillations in the envelope of the autocorrelation (EACF) of the time series, filtered in the expected frequency domain [44, 45]. This approach allows for deriving the mean large separation of a solar-like oscillation spectrum in a blind way without prior information. It has shown to be efficient in cases of low SNR [e.g. 46-48]. The reliability of the result is given by an $H_{0}$ test: when the EACF is above a threshold level, the null hypothesis can be rejected, implying that a signal might have been detected.

The envelope of the autocorrelation displays a maximum in between 1.96 and $2.15 \mathrm{~h}$ in the frequency range $[600,1400] \mu \mathrm{Hz}$ (Fig. 6). From the $H_{0}$ test, the likelihood for this peak to be a signal is about $95 \%$. This would correspond with a large frequency spacing ranging from 258 to $283 \mu \mathrm{Hz}$ if it is the result of Neptune's oscillations. However, if this excess of power in the autocorrelation di- 


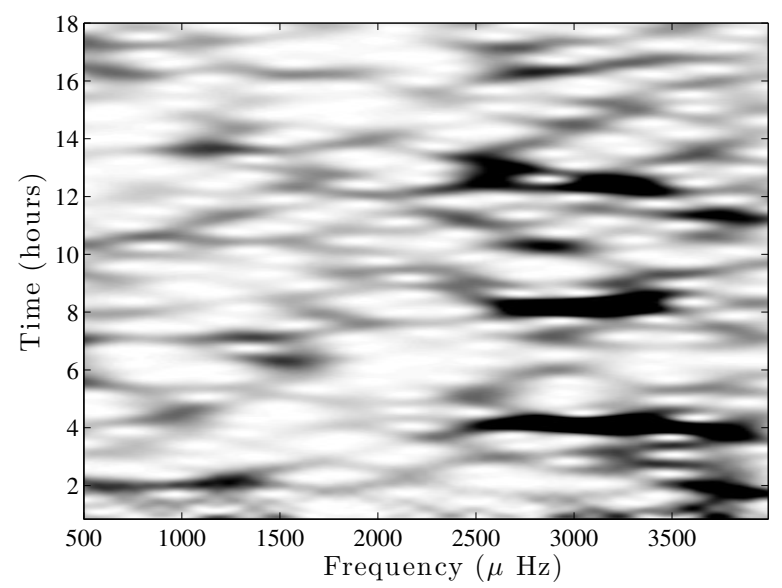

Figure 6: Envelope of the autocorrelation function (EACF) as function of frequency ( $x$-axis) and time ( $y$-axis). The EACF was computed every $20 \mu \mathrm{Hz}$ from 500 to $4000 \mu \mathrm{Hz}$, by filtering the time series in a frequency bandpass of $500 \mu \mathrm{Hz}$. The darker a region is, the larger the correlation. Solar modes are clearly visible in between 2500 and $3500 \mu \mathrm{Hz}$ at approximately 4, 8 and 13 hours. From [43].

agram is generated by Neptune's oscillations, we should find peaks corresponding to its rotation period (16-17h) and some harmonics, as modes are split by it. Figure 6 does not exhibit significant maxima at 16 or 8 hours. As a comparison, we displayed the correlation diagram up to frequencies including the solar oscillations. We clearly detect the Sun's large separation, as well as secondary peaks due to the various overtones and the separation between $\ell=0$ and $\ell=1$ modes. Finally, we investigated whether a structure could be detected in the échelle diagram corresponding with $\mathrm{a} \approx 280 \mu \mathrm{Hz}$ and nothing was observed, making it useless to reproduce in the paper. The signal in the $[600,1400] \mu \mathrm{Hz}$ range does not show similar features, and we conclude this 2 -hour signal it is likely a spurious signal.

\subsection{First Indirect Detection of Solar Oscillations in Intensity Measurements}

The 5-minute oscillations in the Sun are routinely used as a reference point for interpreting solar-like oscillations in other stars. This has become increasingly important with the huge amount of high-quality data being returned by NASA's Kepler Mission. Ideally, the measurements of solar oscillations that act as a reference should be observed with the same instrument as the stars.

Observations of Neptune with K2 allowed for a unique opportunity to measure integrated disk seismic properties of the Sun in reflected light and determine fundamental properties (mass, radius) of the Sun as a distant star. Solar oscillations have been measured in radial velocity from the Moon [49], and also from the blue sky in both equivalent width [50] and radial velocity [51]. To our knowledge, Gaulme et al. [42] analysis of K2 photometric ob-
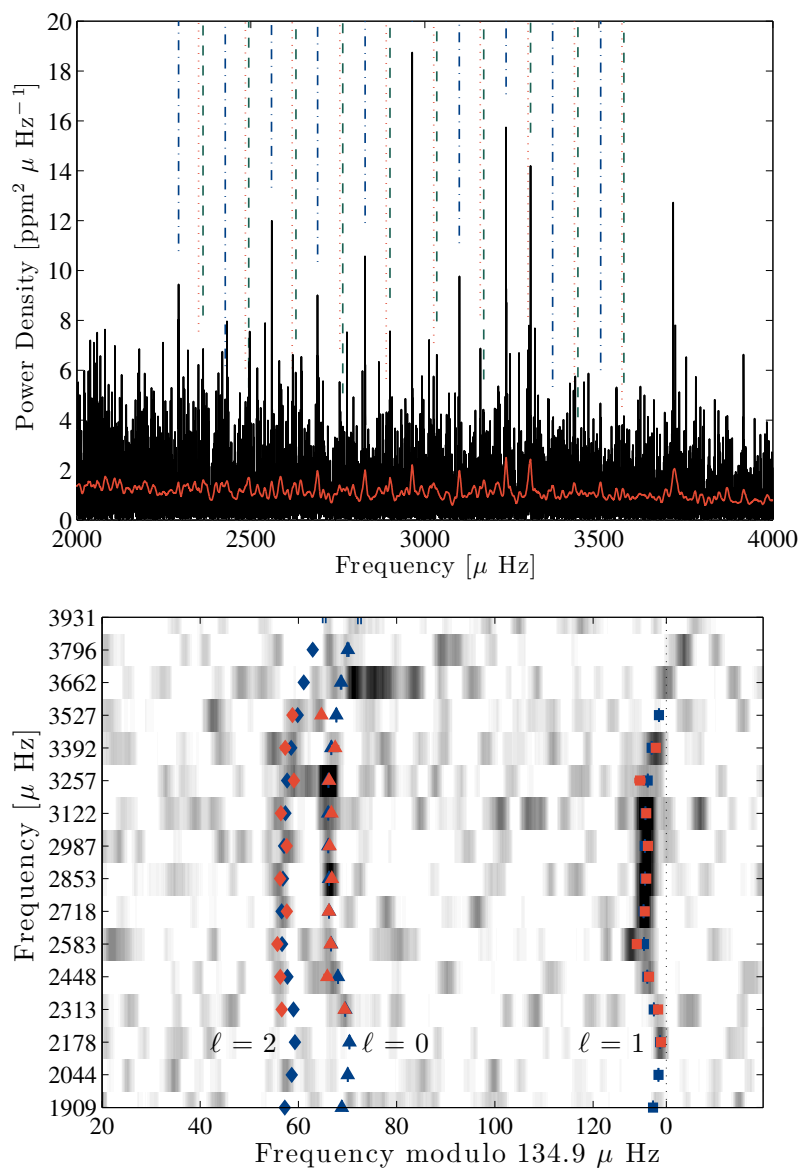

Figure 7: Top panel: K2 Neptune power spectral density on a linear scale $\left(\mathrm{ppm}^{2} \mu \mathrm{Hz}^{-1}\right)$ as a function of frequency $(\mu \mathrm{Hz})$. The dot-dashed lines indicate the frequencies Gaulme et al. detected and modeled with the peak-bagging method. Bottom panel: échelle diagram of the power density spectrum. Darker regions correspond to larger peaks in power density. The power density spectrum is smoothed by a weighted moving average over seven bins and folded into $134.9 \mu \mathrm{Hz}$ chunks; each is then stacked on top of its lower-frequency neighbor. Red dots indicate K2 frequencies and the blue dots BiSON's. Error bars are smaller than symbols. From [42].

servations of reflected solar light from Neptune is the first indirect detection of solar oscillations in intensity.

Another reason for our interest in the $\mathrm{K} 2$ observations of Neptune is to calibrate the amplitudes of oscillations in the Sun. There has been considerable effort towards understanding how the amplitudes of solar-like oscillations vary from the Sun to other stars, both theoretically [5260] and observationally [61-68]. A good measurement of the solar amplitude with Kepler would serve as an important calibration.

The global asteroseismic approach involves measuring $\Delta v$ and $v_{\max }$ from the PSD of the light curve. To determine $v_{\max }$, one must model background noise in the frequency domain, which is typically dominated by the correlated stellar noise (spots, granulation, meso- and supergranulation). In this case, the background variability arises from sources other than solar spots and granulation. This 
is obvious when comparing the K2 PSD with simultaneous SOHO/VIRGO (green channel) data (Fig. 4). K2's background overwhelms VIRGO's by up to four orders of magnitude. K2's noise likely results from residuals of effects associated with Neptune's motion across the detector (pixel response non-uniformity, saturation, pointing jitter, etc). It is unlikely that Neptune atmospheric features are responsible for such a noise level given the planet's smooth aspect in the visible, except for isolated cloud structures that appear as outstanding peaks, as we discussed above.

To minimize the bias in estimating the global asteroseismic parameters, Gaulme et al. measured the global helioseismic parameters and individual mode properties (peak-bagging) independently with slightly different approaches, by several groups of fitters. The idea was to proceed as one would if this target were one of the many oscillating stars detected by Kepler. They obtained a determination of $\Delta v$ that is consistent with measurements from SOHO/VIRGO/SPM and BiSON. Differences of about 2 to $3 \sigma$, depending on methods, were observed in the determination of $v_{\max }$ relative to the usual solar reference $(3100 \mu \mathrm{Hz}$, e.g. [69]). The application of asteroseismic scaling relations produces a mass and radius of $1.14 \pm 0.06 M_{\odot}$ and $1.05 \pm 0.02 R_{\odot}$ for the Sun. However, a close look at the simultaneous photometric VIRGO/SPM data indicates that $v_{\max }$ was actually larger than the usual solar reference $(3160 \pm 10 \mu \mathrm{Hz})$, which is not surprising given the stochastic nature of solar oscillations. By taking into account the SNR, the value of $v_{\max }$ that is measured from $\mathrm{K} 2$ data is consistent with VIRGO, and corresponds to the upper $20 \%$ of the posterior density probability.

The peak-bagging technique leads to mean amplitude and width that match those from VIRGO within error bars. Frequencies are very consistent among fitters and with VIRGO and BiSON. Except for a few peaks with low SNR, all fit within $1 \sigma$. In regards to mode widths, the dispersion is relatively large between fitters, with commonly a factor of two difference, but the error bars are large and mostly overlap. The measured widths from K2 are generally larger than those measured by Gaulme on simultaneous VIRGO data, but agree relatively well with those retrieved from 14 years of VIRGO by Stahn (2010, PhD manuscript) and simultaneous BiSON measurements. As for the amplitudes, mean VIRGO amplitudes $(3.6 \pm 0.3 \mathrm{ppm})$ are larger but still compatible with $\mathrm{K} 2$ $(2.3 \pm 0.5 \mathrm{ppm})$. However, K2's amplitudes are lower than VIRGO's, especially around $v_{\max }$, where VIRGO amplitudes are $\approx 4.5 \mathrm{ppm}$ and $\mathrm{K} 2 \approx 3 \mathrm{ppm}$, i.e. 1/3 larger. This is presumably due to Kepler's broader and, in particular, redder passband. [70] showed the ratio of the mode amplitudes measured from VIRGO data for the three channels are: blue-to-green $\approx 1.4$ and green-to-red $\approx 2$, which is consistent with the discrepancies we observe with respect to VIRGO green channel data. Note that BiSON amplitudes are not directly comparable because it is a velocity measurement.
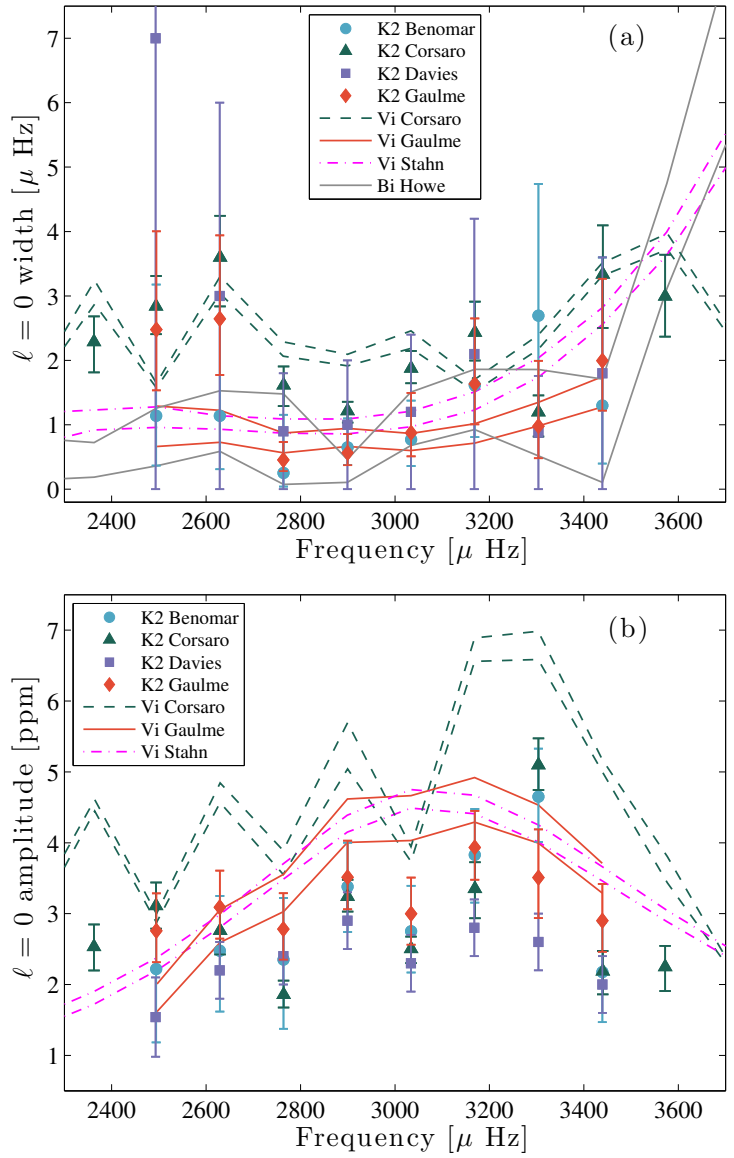

Figure 8: Oscillation width and amplitude as a function of frequency for radial modes. Dashed and dash-dotted lines indicate estimates from VIRGO data (black for simultaneous and magenta for 14 years data). Different colors, symbols and names indicate the results obtained by the different group of fitters from Gaulme et al. (figure extracted from [42]).

\section{Conclusions}

Seismology applied to giant planets needs a clear recording of the oscillation spectrum of any giant planet, where mode frequencies are unambiguously determined. Due to some peculiarities of giant planets with respect to the Sun or stars (resolved objects, wrapped by clouds, fast rotators), a specific instrumentation is required. Gaulme \& Mosser (2005) [35] have explored the possibility of using a space imager as CoRoT for detecting the oscillations of giants planets in the reflected solar flux. They showed that answering to this question depends strongly on the cloud vertical structure and microphysics, which are mostly unknown. The Kepler K2 mission represented a unique opportunity of testing this technique on the two ice giants. It observed Neptune continuously for 49 days starting in December 2015 [38], and did the same with Uranus about a year later for 27 days. Neptune's observations have not brought any detection of Neptune's oscillations, but have allowed for detecting the Sun's oscillations reflected on Neptune [42]. K2 Uranus data are currently being processed and it is too early to reject the detection of planetary oscillations, even though they do not seem to be de- 
tectable. Nevertheless, solar oscillations are detected at a similar SNR level as for Neptune, and we are keenly interested to see what solar $v_{\max }$ the $\mathrm{K} 2$ Uranus data will suggest.

Overall, the best instrumental approach to get good SNR oscillations spectra of giant planets are the same as for the Sun, i.e. Doppler imaging. After first the results of the SYMPA instrument, a team led by FrançoisXavier Schmider (Observatoire de la Côte d'Azur) proposed to place a payload inherited from SYMPA onboard of the JUICE ESA mission to the Jupiter's system. The payload was not selected in the end of a 5-year process, but we were granted enough fundings (CNES, ESA, Thales, NASA) to develop a prototype. The project named JOVIAL (in France) or JIVE (in the US) has now evolved into a three-instrument ground-based network, with sites in France, USA, and Japan, in the same way as helioseismic BiSON, GONG, or asteroseismic SONG. The objective is to observe Jupiter's oscillations and identify its modes up to a degree $\ell=10$, to definitively answer the question of the existence of a rocky core. A single-site test campaign was led in 2015, and the network will be operational in 2018.

\section{References}

[1] G. Magni, A. Coradini, PSS, 52, 343 (2004)

[2] L. Mayer, T. Quinn, J. Wadsley, J. Stadel, Science 298, 1756 (2002)

[3] D. Saumon, T. Guillot, ApJ, 609, 1170 (2004)

[4] B. Militzer, W.B. Hubbard, J. Vorberger, I. Tamblyn, S.A. Bonev, ApJL, 688, L45 (2008)

[5] N. Nettelmann, A. Becker, B. Holst, R. Redmer, ApJ, 750, 52 (2012)

[6] T. Guillot, Science 286, 72 (1999)

[7] P. Gaulme, B. Mosser, F.X. Schmider, T. Guillot, Seismology of Giant Planets (2015), pp. 189-202

[8] N. Nettelmann, R. Helled, J.J. Fortney, R. Redmer, PSS, 77, 143 (2013)

[9] J.J. Fortney, N. Nettelmann, Space Science Reviews, 152, 423 (2010)

[10] D.J. Stevenson, Icarus, 62, 4 (1985)

[11] J. Leconte, G. Chabrier, A\&A, 540, A20 (2012)

[12] R. Helled, T. Guillot, ApJ, 767, 113 (2013)

[13] P. Gaulme, F.X. Schmider, J. Gay, T. Guillot, C. Jacob, A\&A, 531, A104+ (2011)

[14] P.G.J. Irwin, Giant Planets of Our Solar System (2009)

[15] G.F. Lindal, AJ, 103, 967 (1992)

[16] S.V. Vorontsov, V.N. Zharkov, V.M. Lubimov, Icarus 27, 109 (1976)

[17] D. Bercovici, G. Schubert, Icarus 69, 557 (1987)

[18] D. Deming, M.J. Mumma, F. Espenak, D.E. Jennings, T. Kostiuk, G. Wiedemann, R. Loewenstein, J. Piscitelli, ApJ, 343, 456 (1989)

[19] F.X. Schmider, E. Fossat, B. Mosser, A\&A, 248, 281 (1991)
[20] B. Mosser, D. Mekarnia, J.P. Maillard, J. Gay, D. Gautier, P. Delache, A\&A, 267, 604 (1993)

[21] B. Mosser, J.P. Maillard, D. Mékarnia, Icarus 144, 104 (2000)

[22] C.M. Walter, M.S. Marley, D.M. Hunten, A.L. Sprague, W.K. Wells, A. Dayal, W.F. Hoffmann, M.V. Sykes, L.K. Deutsch, G.G. Fazio et al., Icarus, 121, 341 (1996)

[23] B. Mosser, P. Galdemard, P. Lagage, E. Pantin, M. Sauvage, P. Lognonne, D. Gautier, F. Billebaud, T. Livengood, H.U. Kaufl, Icarus 121, 331 (1996)

[24] F.X. Schmider, J. Gay, P. Gaulme, C. Jacob, L. Abe, M. Alvarez, J.A. Belmonte, E. Fossat, B. Gelly, T. Guillot et al., A\&A, 474, 1073 (2007)

[25] J.W. Harvey, F. Hill, R.P. Hubbard, J.R. Kennedy, J.W. Leibacher, J.A. Pintar, P.A. Gilman, R.W. Noyes, A.M. Title, J. Toomre et al., Science 272, 1284 (1996)

[26] P.H. Scherrer, R.S. Bogart, R.I. Bush, J.T. Hoeksema, A.G. Kosovichev, J. Schou, W. Rosenberg, L. Springer, T.D. Tarbell, A. Title et al., Sol. Phys., 162, 129 (1995)

[27] P.H. Scherrer, J. Schou, R.I. Bush, A.G. Kosovichev, R.S. Bogart, J.T. Hoeksema, Y. Liu, T.L. Duvall, J. Zhao, A.M. Title et al., Sol. Phys., 275, 207 (2012)

[28] P. Gaulme, F.X. Schmider, J. Gay, C. Jacob, M. Alvarez, M. Reyes-Ruiz, J.A. Belmonte, E. Fossat, F. Jeanneaux, J.C. Valtier, A\&A, 490, 859 (2008)

[29] M.S. Marley, C.C. Porco, Icarus, 106, 508 (1993)

[30] M.M. Hedman, P.D. Nicholson, AJ, 146, 12 (2013)

[31] J. Fuller, Icarus, 242, 283 (2014)

[32] M.S. Marley, Icarus, 234, 194 (2014)

[33] P.G. Beck, T.R. Bedding, B. Mosser, D. Stello, R.A. Garcia, T. Kallinger, S. Hekker, Y. Elsworth, S. Frandsen, F. Carrier et al., Science 332, 205 (2011)

[34] B. Mosser, A\&A, 293, 586 (1995)

[35] P. Gaulme, B. Mosser, Icarus 178, 84 (2005)

[36] E. Michel, A. Baglin, M. Auvergne, C. Catala, R. Samadi, F. Baudin, T. Appourchaux, C. Barban, W.W. Weiss, G. Berthomieu et al., Science 322, 558 (2008)

[37] W.J. Borucki, D. Koch, G. Basri, N. Batalha, T. Brown, D. Caldwell, J. Caldwell, J. ChristensenDalsgaard, W.D. Cochran, E. DeVore et al., Science 327, 977 (2010)

[38] A.A. Simon, J.F. Rowe, P. Gaulme, H.B. Hammel, S.L. Casewell, J.J. Fortney, J.E. Gizis, J.J. Lissauer, R. Morales-Juberias, G.S. Orton et al., ApJ, 817, 162 (2016)

[39] S.C. Martin, I. de Pater, P. Marcus, Ap\&SS, 337, 65 (2012)

[40] E. Karkoschka, Icarus, 215, 439 (2011)

[41] L.A. Sromovsky, Science 254, 684 (1991)

[42] P. Gaulme, J.F. Rowe, T.R. Bedding, O. Benomar, E. Corsaro, G.R. Davies, S.J. Hale, R. Howe, R.A. Garcia, D. Huber et al., ApJL, 833, L13 (2016) 
[43] J.F. Rowe, P. Gaulme, J.J. Lissauer, M.S. Marley, A.A. Simon, H.B. Hammel, V. Silva Aguirre, T. Barclay, O. Benomar, P. Boumier, Submitted to ApJL (2016)

[44] I.W. Roxburgh, S.V. Vorontsov, MNRAS, 369, 1491 (2006)

[45] B. Mosser, T. Appourchaux, A\&A, 508, 877 (2009)

[46] B. Mosser, E. Michel, T. Appourchaux, C. Barban, F. Baudin, P. Boumier, H. Bruntt, C. Catala, S. Deheuvels, R.A. García et al., A\&A, 506, 33 (2009)

[47] P. Gaulme, S. Deheuvels, W.W. Weiss, B. Mosser, C. Moutou, H. Bruntt, J. Donati, M. Vannier, T. Guillot, T. Appourchaux et al., A\&A, 524, A47+ (2010)

[48] B. Mosser, K. Belkacem, M.J. Goupil, A. Miglio, T. Morel, C. Barban, F. Baudin, S. Hekker, R. Samadi, J. De Ridder et al., A\&A, 517, A22 (2010)

[49] H. Kjeldsen, T.R. Bedding, R.P. Butler, J. Christensen-Dalsgaard, L.L. Kiss, C. McCarthy, G.W. Marcy, C.G. Tinney, J.T. Wright, ApJ, 635, 1281 (2005)

[50] H. Kjeldsen, T.R. Bedding, M. Viskum, S. Frandsen, AJ, 109, 1313 (1995)

[51] H. Kjeldsen, T.R. Bedding, T. Arentoft, R.P. Butler, T.H. Dall, C. Karoff, L.L. Kiss, C.G. Tinney, W.J. Chaplin, ApJ, 682, 1370 (2008)

[52] J. Christensen-Dalsgaard, S. Frandsen, Sol. Phys., 82, 469 (1983)

[53] H. Kjeldsen, T.R. Bedding, A\&A, 293, 87 (1995)

[54] H. Kjeldsen, T.R. Bedding, A\&A, 529, L8 (2011)

[55] G. Houdek, N.J. Balmforth, J. ChristensenDalsgaard, D.O. Gough, A\&A, 351, 582 (1999)

[56] G. Houdek, D.O. Gough, MNRAS, 336, L65 (2002)

[57] G. Houdek, Stochastic excitation and damping of solar-like oscillations, in Proceedings of SOHO 18/GONG 2006/HELAS I, Beyond the spherical Sun (2006), Vol. 624 of ESA Special Publication, p. 28.1

[58] R. Samadi, D. Georgobiani, R. Trampedach, M.J. Goupil, R.F. Stein, A. Nordlund, A\&A, 463, 297
(2007)

[59] R. Samadi, K. Belkacem, M.A. Dupret, H.G. Ludwig, F. Baudin, E. Caffau, M.J. Goupil, C. Barban, A\&A, 543, A120 (2012)

[60] K. Belkacem, R. Samadi, M.J. Goupil, Journal of Physics Conference Series 271, 012047 (2011)

[61] R. Samadi, H.G. Ludwig, K. Belkacem, M.J. Goupil, O. Benomar, B. Mosser, M.A. Dupret, F. Baudin, T. Appourchaux, E. Michel, A\&A, 509, A16 (2010)

[62] D. Huber, T.R. Bedding, D. Stello, S. Hekker, S. Mathur, B. Mosser, G.A. Verner, A. Bonanno, D.L. Buzasi, T.L. Campante et al., ApJ, 743, 143 (2011)

[63] D. Huber, T.R. Bedding, T. Arentoft, M. Gruberbauer, D.B. Guenther, G. Houdek, T. Kallinger, H. Kjeldsen, J.M. Matthews, D. Stello et al., ApJ, 731, 94 (2011)

[64] T.L. Campante, R. Handberg, S. Mathur, T. Appourchaux, T.R. Bedding, W.J. Chaplin, R.A. García, B. Mosser, O. Benomar, A. Bonanno et al., A\&A, 534, A6 (2011)

[65] W.J. Chaplin, H. Kjeldsen, T.R. Bedding, J. Christensen-Dalsgaard, R.L. Gilliland, S.D. Kawaler, T. Appourchaux, Y. Elsworth, R.A. García, G. Houdek et al., ApJ, 732, 54 (2011)

[66] K. Belkacem, M.A. Dupret, F. Baudin, T. Appourchaux, J.P. Marques, R. Samadi, A\&A, 540, L7 (2012)

[67] B. Mosser, Y. Elsworth, S. Hekker, D. Huber, T. Kallinger, S. Mathur, K. Belkacem, M.J. Goupil, R. Samadi, C. Barban et al., A\&A, 537, A30 (2012)

[68] E. Corsaro, H.E. Fröhlich, A. Bonanno, D. Huber, T.R. Bedding, O. Benomar, J. De Ridder, D. Stello, MNRAS, 430, 2313 (2013)

[69] A.M. Broomhall, W.J. Chaplin, G.R. Davies, Y. Elsworth, S.T. Fletcher, S.J. Hale, B. Miller, R. New, MNRAS, 396, L100 (2009)

[70] A. Jiménez, T. Roca Cortés, G. Severino, C. Marmolino, ApJ, 525, 1042 (1999) 